\title{
Disordered environments in spatial games
}

\author{
Mendeli H. Vainstein and Jeferson J. Arenzon* \\ Instituto de Física Universidade Federal do Rio Grande do Sul, Caixa Postal 15051, 91501-970 Porto Alegre, RS - Brazil
}

(Received 4 April 2001; published 22 October 2001)

\begin{abstract}
The Prisoner's dilemma is the main game theoretical framework in which the onset and maintainance of cooperation in biological populations is studied. In the spatial version of the model, we study the robustness of cooperation in heterogeneous ecosystems in spatial evolutionary games by considering site diluted lattices. The main result is that, due to disorder, the fraction of cooperators in the population is enhanced. Moreover, the system presents a dynamical transition at $\rho^{*}$, separating a region with spatial chaos from one with localized, stable groups of cooperators.
\end{abstract}

DOI: 10.1103/PhysRevE.64.051905

PACS number(s): 87.23.Cc, 05.50.+q

\section{INTRODUCTION}

Game theoretical methods have been applied, in a quite successful way, to several different fields $[1,2]$, biology being one of the most successful branches [3-6]. In these evolutionary games, rewards are translated in terms of subsequent reproductive success, a more natural and clear scale than the rationality dependent ones used in, for example, economy. Of particular interest is the emergence and sustainability of cooperation, which has attracted a lot of attention as it poses a difficult problem from an evolutionary point of view since cooperators, by their nature, have their fitness decreased while interacting with defectors. Theories advanced to explain the evolution of cooperation usually consider either kin selection [5], reciprocal altruism [7,8], or group selection [9-13], the separation between them not always being clear. Group foraging and young raising [14], alarm calls $[15,16]$, bacteria-infecting viruses [17], predator inspection by fish [18], bird female-female cooperation [19], and cleaner fish [20], are but a few examples of such acts in biological populations, ranging from simple to complex organisms. A number of other examples may be found in Ref. [6].

The Prisoner's dilemma game is generally studied as an archetypical model for reciprocal altruism. Each of the two players either cooperates $(C)$ or defects $(D)$, without knowledge of the opponent's strategy. The result depends on the mutual choice, and is given by the payoff matrix whose elements are a reward $R$ (punishment $P$ ) if both cooperate (defect), $S$ (sucker's payoff), and $T$ (temptation) if one cooperates and the other defects, respectively. Moreover, these quantities should satisfy the inequalities $T>R>P>S$ and $2 R>T+S$. It is clear that, independent of the opponent's choice, defecting is always the best bet. Thus two basic evolutionary problems are (i) the onset of cooperation, that is, under which conditions a given cooperative behavior can invade a population of defectors and, once established, (ii) its stability, that is, under which conditions the population of cooperators is uninvadable. In other words, we are looking for evolutionary stable strategies (ESS's) [3,4,21] where there is stable mutual cooperation between individuals. In a

\footnotetext{
*Email address: arenzon@if.ufrgs.br
}

random mating, infinite population of asexual (haploid) elements, where two pure strategies are present (cooperators $C$ and defectors $D$ ), it can be shown that defecting will be the most rewarding strategy (an ESS). If instead of only interacting once or a known finite number of times, the players have a probability $w>0$ of meeting again in the next round and remember the chosen strategies in previous encounters, more complex rules may be devised [1]. For instance, in a round robin tournament, Axelrod [1] found that tit-for-tat (TFT) (starts cooperating and, after that, do what the opponent did in the previous step) was the most successful strategy, but other successful strategies were also found [22]. Nevertheless, TFT cannot invade a population of defectors before reaching a minimum population, which can be achieved in several ways $[8,23,24]$.

Following a suggestion of Axelrod [1], Nowak and coworkers [25-27] showed how cooperation can arise even with only pure, memoryless strategies, such as "always cooperate" or "always defect" in the presence of spatial structure. They considered a deterministic cellular automaton consisting of a square lattice with near and next nearest neighbor interactions and self-interaction. Since the agents are spatially localized, they are more likely to interact only with their nearest neighbors, differently from the standard, mean-field-like approach, that considers an infinite, random mixing population. The reasons for this are manifold: individuals usually occupy well-defined territorial regions, individuals do not move far from their places of birth (population viscosity [5]), interactions occur in places where animals usually meet such as water ponds, etc. After combat, each player compares its total payoff with the ones of its neighbors and changes strategy, following the strategy with the greatest payoff. For a certain range of values of the payoff matrix, very complex spatial patterns show up with cooperators and defectors coexisting (spatial chaos). In these structured populations, cooperative strategies can build clusters in which the benefits of mutual cooperation can outweigh losses against defectors, maintaining the population of cooperators to be stable. Irrespective of the initial state that may be chosen with either only one initial defector or a fraction of randomly distributed defectors, the asymptotic density depends only on the payoff matrix parameters. The actual values depend on the neighborhood chosen for the dynamics, and on whether self-interactions are included or not $[25,26]$. 
This cooperation enhancement effect due to the spatial structure is also seen in other games. For instance, in the spatial version of the Hawk-Dove game [26,28,29], the density of doves (analogous to cooperative individuals) is increased by the spatial structure, although in this game polymorphism would exist even in the absence of spatial effects. In a similar way, several strategies are more successful in a spatially structured population than otherwise [30,31].

In real populations, however, not all individuals interact the same number of times, either due to the nonsynchronous character of the interaction or to the environmental structure that prevents some of the contacts. Both spatial and temporal aspects of the environment affect the interactions between individuals, being central issues in ecological and evolutionary theory. Thus a natural question arises: how is cooperation affected by this inherent inhomogeneity? In other words, how robust is cooperation in the presence of disorder? In this paper we apply a simple approach to this question by considering a regular lattice where some sites are empty. Dilution can be either quenched (fixed) or annealed (evolving). In the latter case, individuals may diffuse in the lattice, which will be considered in detail in a future work. Quenched vacancies (or defects) may account for the presence of environmental features (e.g., geographical) in the game, causing some individuals to have fewer neighbors than others. Deviations from the ordered lattice can also be achieved in several other ways, for instance, by allowing that some of the individuals also interact with distant ones, in a small-world network fashion [32,33]. In Refs. [27,34], a diluted lattice was used with individuals interacting inside a region of radius $r$, and a persistent polymorphism of $C$ 's and $D$ 's was found, unless $r$ was made too large, reaching the long range connectivity associated with mean field behavior, where the defector population dominates. Nevertheless, their treatment was quite brief and several interesting dynamical behaviors passed unnoticed, as well as the important issue of whether or not disorder enhances the fraction of cooperators in the population.

Here we show that, depending on the amount of disorder, cooperation can be enhanced, there being a point where a dynamical transition settles in, separating the region with spatial chaos from the one with localized groups of cooperators. The paper is organized as follows. In Sec. II, a diluted version of the spatial Prisoner's game is presented and the order parameters are defined. In Sec. III the main results are presented. Finally, in Sec. IV, we present conclusions and comments.

\section{MODEL}

We consider the spatial version of the Prisoner's dilemma [1,25-27,30,34-37], placing the interacting elements in the vertices of a $d$-dimensional array, usually a hypercube, with periodic boundary conditions. The results presented here are for $d=2$. Differently from the original case, we allow that some of the sites may be empty. To describe the occupation of the $i$ site $\left(1<i<N=L^{d}\right.$, where $L$ is the system linear dimension) we take $n_{i}$ to be either 1 , if the site is occupied, or 0 otherwise. In every generation, each individual assumes an unchangeable strategy from a set $\Omega$ and, following Ref. [25], we only consider the simplest case of pure strategies $C$ and $D$, represented by the variable $S_{i}= \pm 1$, respectively. In each step (generation), the $i$ th individual $\left(n_{i} \neq 0\right)$ combats with all other elements inside a given neighborhood $\mathcal{V}_{i}$, and accumulates a payoff $p_{i}$, depending on the chosen strategies, according to the reduced payoff table for the Prisoner's game [25]: $R=1, P=S=0$, and $T=b>1$, reducing the problem to only one parameter (besides the density). For the case considered here, where the neighborhood $\mathcal{V}_{i}$ is restricted to nearest neighbors of site $i$ and no self-interactions are included, both $C$ and $D$ coexist in the region $4 / 3<b<3 / 2$ in which also the number of active sites (see below) is large. For $1<b$ $<4 / 3$ cooperators dominate while for $b>3 / 2$, defectors are dominant. The player's payoff is a measure of its reproductive success: when reproducing, the $i$ th element compares its own payoff with all $j \in \mathcal{V}_{i}$ and changes to the strategy of the site that has the greatest payoff in $\{i\} \cup \mathcal{V}_{i}$. In this way, the global density is kept fixed since no empty site will be ever filled.

To characterize the macroscopic behavior of the system we introduce two order parameters. Let $\rho_{c}(t)$ represent the fraction of cooperators at a given time,

$$
\rho_{c}(t)=\frac{1}{2 N} \sum_{i=1}^{N}\left(S_{i}+1\right) n_{i}
$$

where $N=L^{d}$ is the total number of sites. Clearly, $\rho_{d}(t)$, the defectors' density, is $\rho_{d}(t)=\rho-\rho_{c}(t)$, where $\rho$ is the total density. Since we are interested in the long time regime, and the results depend on the choice of the frozen empty sites, we define the order parameter as the average over time $(\langle\cdots\rangle)$ and over the realizations of the disorder $(\bar{\cdots})$ of the asymptotic cooperators density $\rho_{c}=\left\langle\overline{\rho_{c}(\infty)}\right\rangle$ for large $N$. Sometimes it is more useful to have the relative cooperators' density $\rho_{c} / \rho$. Thus $\rho_{c}=0$ means that the population was fully invaded by defectors, and $\rho_{c}=\rho$ that it was invaded by cooperators. An intermediate case $0<\rho_{c}<\rho$, in which both strategies coexist, is also possible. Moreover, it is interesting to know the fraction of active sites, that is, the fraction of elements that change strategy in time:

$$
\rho_{a}(t)=\frac{1}{2 N} \sum_{i=1}^{N}\left(1-S_{i}^{t} S_{i}^{t-1}\right) n_{i} .
$$

This defines our second order parameter $\rho_{a}=\left\langle\overline{\rho_{a}(\infty)}\right\rangle$. Thus $\rho_{a}=0$ means that all sites are frozen, and $\rho_{a}=\rho$ means that all elements are changing strategy.

\section{RESULTS}

In Fig. 1, the asymptotic cooperator density $\rho_{c} / \rho$ is plotted against the total occupation of the lattice, $\rho$. If the occupation fraction is near zero, almost all sites are isolated and do not change strategy since there is no combat at all; the asymptotic density is the same as the initial one: $\rho_{c}=\rho_{c}(0)$ $=\rho / 2$. Indeed, for small $\rho$, the curves for different values of $b$ merge. As the density increases, the probability of occur- 


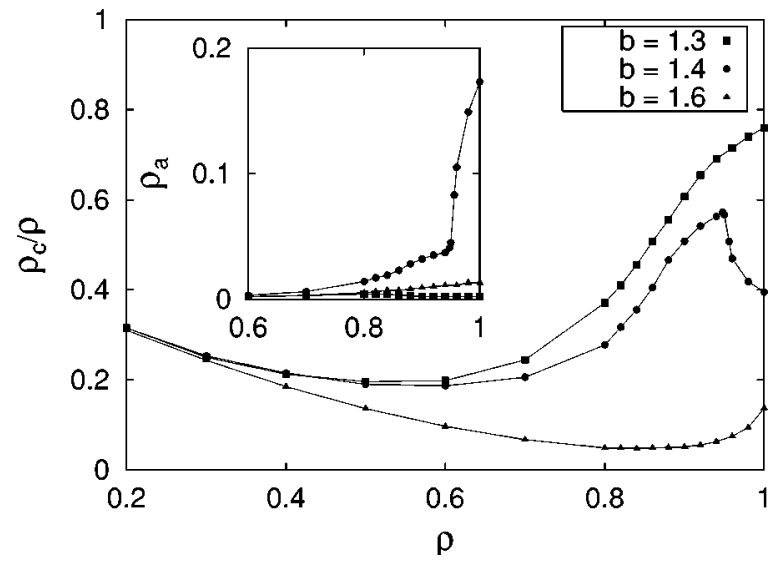

FIG. 1. Asymptotic fraction of cooperators $\rho_{c} / \rho$ as a function of the lattice occupation $\rho$ for several values of $b$. The initial state has $\rho / 2$ cooperators, the lattice size is $L=100$, and averages are taken over 100 samples.

ring pairs of occupied sites increases and, irrespective of the value of $b$, all $C D$ pairs will become $D D$, which can be seen as a decreasing curve of $\rho_{c}$ from the origin. Around $\rho \simeq 0.3$, as the number of interacting individuals increases due to increasing cluster sizes, the dynamics will define the fate of each cluster and the curves for different values of $b$ will depart: the higher $b$ is, the better for defectors and the lower the corresponding cooperator curve. Still further, when clusters of cooperators have a reasonable probability to form, their density starts to increase reobtaining the Novak-May results as the total density approaches unity[25]. The most interesting case occurs if $b$ is in the active region, $4 / 3<b$ $<3 / 2$, where the behavior is not monotonic and a sharp decrease in $\rho_{c}$ appears near $\rho^{*} \simeq 0.95$. At the same point, the fraction of active sites presents a sharp increase, as can be seen in the inset of Fig. 1. Note that for the other regions, the behavior is almost uniform, with a very small number of active sites. Interestingly enough, depending on the region of $b$, the optimum density that maximizes the fraction of cooperators occurs at different values: for $b<4 / 3$, the more individuals the better, and their maximum occurs at $\rho=1$; above $b=3 / 2$, on the other hand, the less occupied the network the better, due to the exploitation by defectors; the maximum occurs in the limit $\rho \rightarrow 0$. In these cases cooperators and defectors, respectively, are at an advantage when interacting. In the intermediate, active region, the behavior is nontrivial, and the maximum occurs at the transition point $\rho^{*}$. Remarkably, this point is much higher than the site percolation transition, which for a square lattice is located at $\rho_{\text {perc }} \simeq 0.59$ [38]: although there is a connected, infinite cluster, regions of active sites are bounded (pinned) to small regions due to the presence of defects, as will be shown below. Below the transition, the approach to equilibrium is exponentially fast, $\rho_{c}(\infty)-\rho_{c}(t) \sim \exp (-t / \tau), \tau$ diverging as one approaches the point $\rho^{*}$. This is because most of the interactions occur inside localized groups. Also, if the lattice is completely filled, the approach is fast. On the other hand, just above the critical value, the presence of defects, and the fact that only some of the groups are depinned, makes the approach to equilibrium

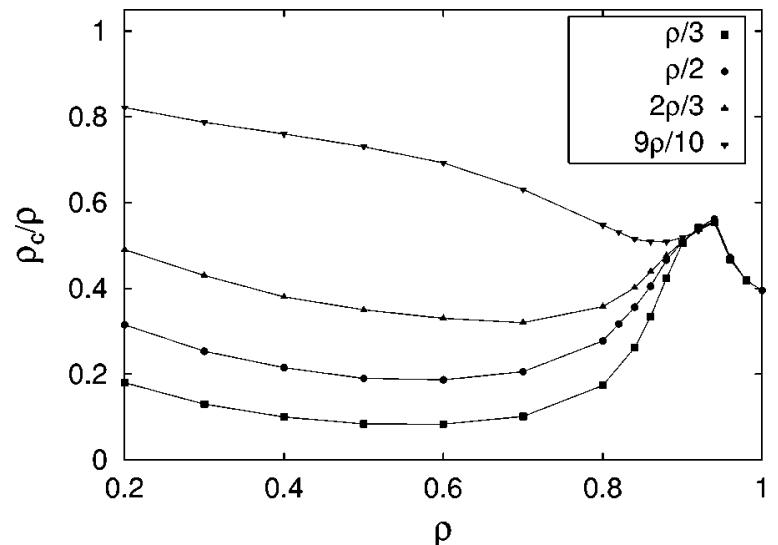

FIG. 2. The asymptotic fraction of cooperators for several different initial densities of cooperators: $L=100$ and $b=1.4$.

extremely slow, power law like, reminiscent of disordered systems.

In the active region, for different initial concentrations of cooperators, it can be seen that almost up to $\rho^{*}$, the asymptotic density depends on the initial state, as shown in Fig. 2. For small $\rho$, since the clusters are independent, obviously the higher the initial fraction of cooperators the higher $\rho_{c}$ will be. Note that the optimum density for cooperation is a function of the initial density of cooperators: for initial concentrations below the height of the peak at $\rho^{*}, \rho_{c}(0)$ $\lesssim 0.54 \rho, \rho^{*}$ is the ideal total concentration for cooperators, while above the smaller $\rho$ is the better. Slightly below $\rho^{*}$ all curves merge because the cluster boundaries are no longer completely pinned, and the memory of the initial state is washed out.

Although both cooperators and active site densities are useful parameters to describe the system asymptotic behavior, they are not sufficient to understand the complex dynamical behavior that arises in the presence of disorder. To clarify what is going on at the transition point, we also measured the persistence, the fraction $P\left(t, t_{w}\right)$ of sites that do not change strategy between an initial waiting time $t_{w}$, and the time $t>t_{w}$ [39-42], as can be seen in Figs. 3 and 4. Different from the fraction of active sites, the persistence is a very complex measure that depends on the whole time history of the system since $t_{w}$. For example, if the persistence does not go to zero, we know that there is a fraction of sites that flip only finitely many times $[43,44]$ (blocking), and domain wall movements are constrained (pinning). That is precisely what happens for $\rho<\rho^{*}$, as shown in Fig. 3: after an initial decrease, the persistence attains, for large times, a plateau whose value depends both on $\rho$ and $t_{w}$. Denoting this plateau by $P\left(\infty, t_{w}\right)$, we note that it goes to zero at $\rho^{*}$, as shown in the inset of Fig. 3 for $t_{w}=0$, signaling a depinning transition. In the critical region, the behavior is power law-like $P(\infty, 0) \sim\left(\rho^{*}-\rho\right)^{2.2}$. It is important to note that the contribution from isolated sites to the plateau is small, and most of the sites forming the plateau come from the infinite cluster. At $\rho^{*}$ and above, the interfaces are no longer constrained; the number of active sites suffer a sudden increase and all sites eventually change strategy. It was shown for several models $[44,45]$ that in the presence of disorder (but also in 


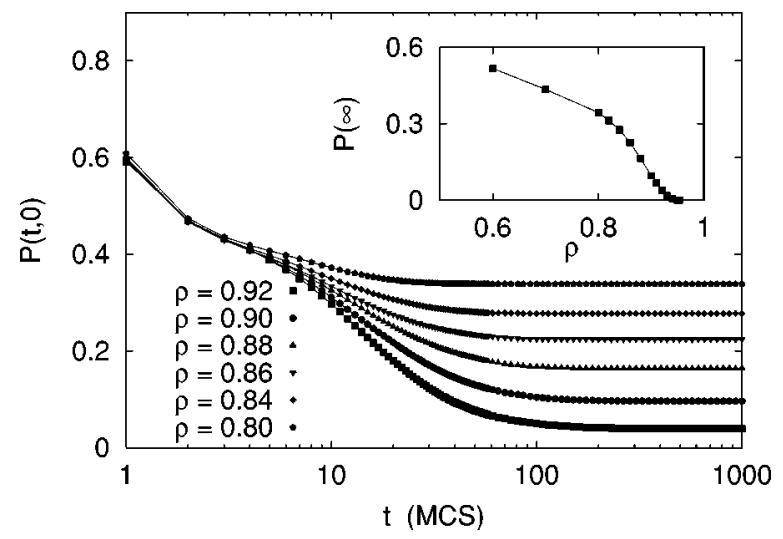

FIG. 3. Persistence $P(t, 0)$ for $L=100, b=1.4$, and several total densities $\rho<\rho^{*}$ as a function of time (measured in Monte Carlo steps). Note that these densities are far above the percolation threshold. As $t \rightarrow \infty$, all curves attain the $\rho$-dependent plateaus $P(\infty, 0)$, shown in the inset as a function of the lattice occupation. The behavior of $P(\infty, 0)$ near $\rho^{*}$ is power law, with an exponent of approximately 2.2 .

some homogeneous systems), the decay to the plateau, $P(t, 0)-P(\infty, 0)$, is exponential $[44,45]$ at large times. The same behavior is found here for a non-Hamiltonian model. In Fig. 5 we present a snapshot of the system below $\rho<\rho^{*}$, showing both empty and pinned sites as well as their strategies. This configuration is almost stable; many of the cooperator groups shown no longer change, and the small amount of active sites is either confined to a few regions or belongs to one of the blinkers present. This has to be compared with the spatial chaos region above $\rho^{*}$, where there is no blocking and every spin flips infinitely many times. Since we are near the transition, the quantity of pinned sites is quite reduced.

For densities $\rho>\rho^{*}$, considering only elements that be-

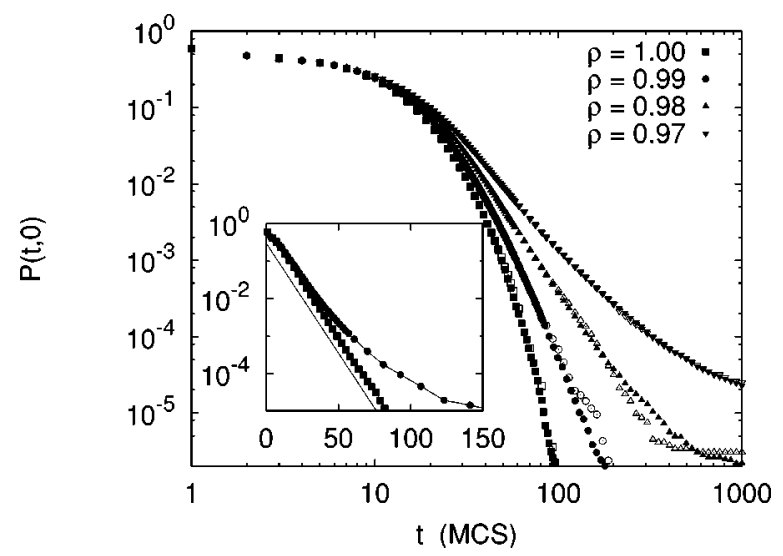

FIG. 4. Persistence $P(t, 0)$ for $L=200$ (empty sites) and 500 (filled ones), $b=1.4$, and several total densities $\rho>\rho^{*}$ as a function of time (measured in Monte Carlo steps). Different from the $\rho$ $<\rho^{*}$ case, here the persistence goes to zero (taking into account only sites in the infinite cluster). For $\rho=1$ it vanishes exponentially (see the semilog plot in the inset), while near this point an initial exponential decay can be seen. Below $\rho=1$, the longtime behavior follows a power law, unless we are too close to $\rho^{*}$, where a crossover behavior to the plateaus is noticed.
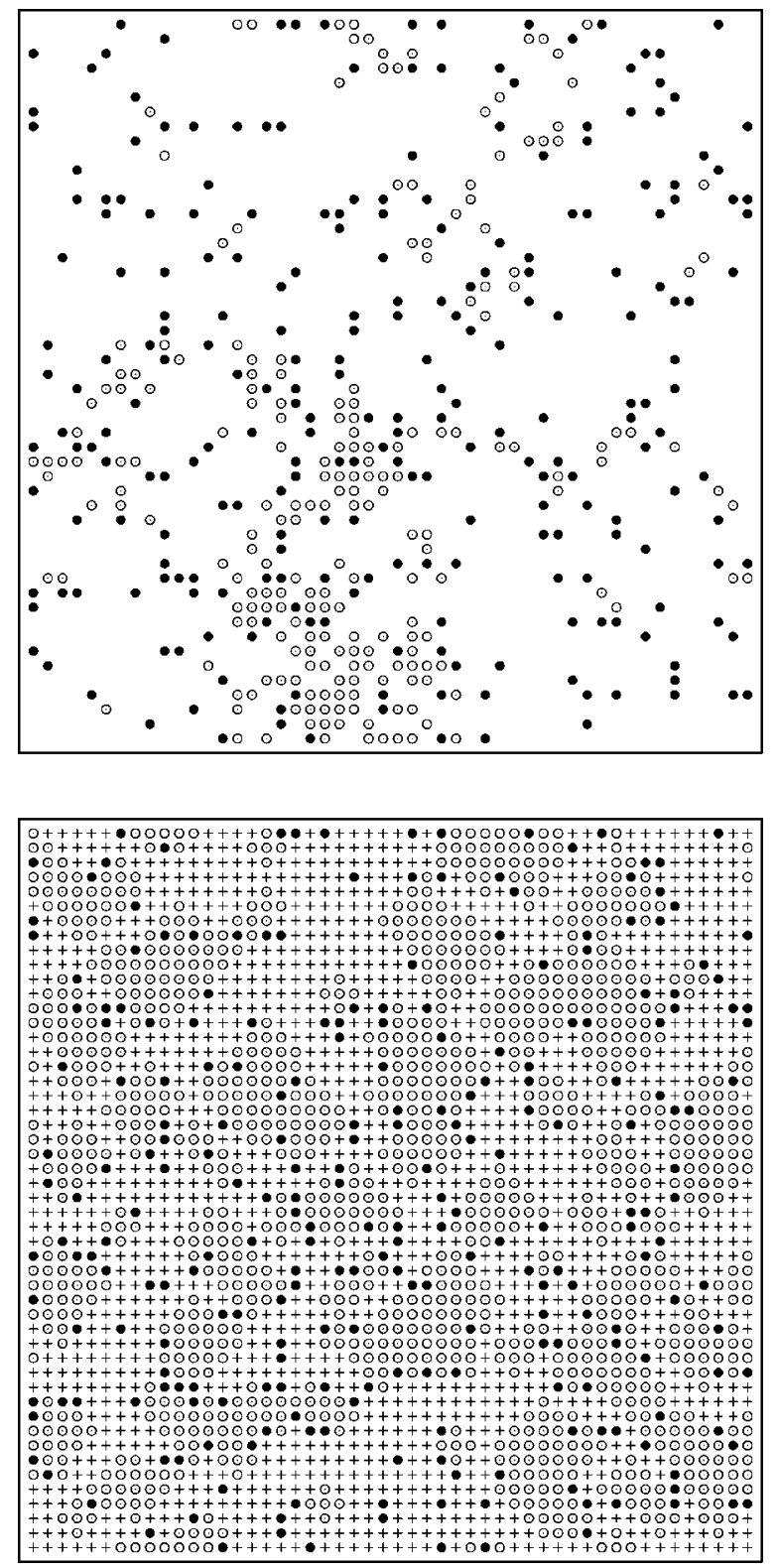

FIG. 5. Snapshot showing the sites that have not changed strategy after $10^{5}$ steps for $L=50$ and total density 0.9 (top). The figure at the bottom shows, at the same time, the cooperators (empty circles) and defectors (crosses). The black points are the empty sites. Those sites that are blocked will remain blocked forever.

long to the infinite cluster, the persistence goes to zero, as shown in Fig. 4. For $\rho=1$, the behavior is exponential, as shown in the inset of Fig. 4. Also note, in the inset (for $\rho$ $=0.99$ ), that as we dilute the lattice, only the initial behavior is exponential. After this initial exponential decrease, whose length decreases as $\rho$ departs from 1, the behavior follows a power law $P(t, 0) \sim t^{-\theta(\rho)}$, and as we approach $\rho^{*}$ from above, the plateau starts to develop. The exponent $\theta(\rho)$ is the persistence exponent and has nontrivial values depending on the total density. For example, $\theta(0.98) \simeq 3.6$ and $\theta(0.99) \simeq 5.7$.

Below the critical value, groups of cooperators are localized and their borders cannot move because of the presence 
of the defects. We say that the groups are pinned by the environment. This explains why the fraction of cooperators is larger in this regime. As the number of defects decreases, the groups start to become depinned; the interfaces start moving and interference effects between the groups appear, explaining the sudden increase in the number of active sites. From the persistence data, we can see that below the critical value, there is a constant fraction of sites in the infinite cluster that never changes strategy, meaning that the clusters are localized. As we approach the depinning transition, some of the groups are depinned and the persistence decreases. Since some of the groups are still pinned, we observe a constant plateau that decreases as $\rho$ approaches $\rho^{*}$. Note that below the transition, the number of active sites is not zero, but small, due to blinking sites.

\section{CONCLUSIONS}

One of the main features of the Prisoner's dilemma, responsible for its widespread use in the problem of cooperation, is its robustness. Here we have shown that the system is able to sustain cooperation even under the presence of lattice disorder, which was already suggested in Ref. [27]. However, what had been unnoticed was the dynamical transition as a function of the amount of quenched disorder as well as the fact that the disorder may enhance cooperation. Dilution changes the scenario presented by Nowak and May [25] for the filled lattice in a dramatic way. The spatial chaos is absent when the disorder is above a given amount, which is reflected by the small number of active sites and the pinning of the interfaces. It must be noted that even without disorder, we are not dealing with a system presenting coarsening as the movement of the interfaces is not ruled by surface tension as, e.g., in the Ising model.

Besides presenting a dynamical transition in the presence of disorder, dilution can enhance the fraction of cooperators in the population. Indeed, in the region $4 / 3<b<3 / 2$, the relative density of cooperators in the population can reach a value almost $40 \%$ higher than in the undiluted case, the maximum occurring at the transition point.

Some questions, however, are still open. For example, if the condition of strong population viscosity is relaxed, disorder is no longer quenched, and diffusion is allowed, what happens to the cooperator groups? Obviously, the results depend on the chosen rule for the diffusing elements, and there are several biologically motivated rules. Work is in progress in this direction. Moreover, from the point of view of the study of persistence, it would be interesting to study the behavior of $P\left(t, t_{w}\right)$ above $\rho^{*}$, for $1 \ll t_{w} \ll t$ (instead of $t_{w}$ $=0)$, as well as to compare the results presented here with the site diluted Ising model.

\section{ACKNOWLEDGMENTS}

We are grateful to H. Chaté, N. Lemke, and L. Peliti for discussions and suggestions, and Y. Levin for a critical reading of the manuscript. Work was partially supported by CNPq and PROPESQ-UFRGS.
[1] R. Axelrod, The Evolution of Cooperation (BasicBooks, New York, 1984).

[2] R. Duncan Luce and H. Raiffa, Games and Decisions (Dover, New York, 1985).

[3] J. Maynard Smith, Evolution and the Theory of Games (Cambridge University Press, Cambridge, UK, 1982).

[4] J. M. Weibull, Evolutionary Game Theory (MIT Press, Cambridge, MA, 1995).

[5] W. D. Hamilton, J. Theor. Biol. 7, 1 (1964).

[6] Game Theory and Animal Behavior, edited by L. A. Dugatkin and H. Reeve (Oxford University Press, New York, 1998).

[7] R. L. Trivers, Q. Rev. Biol. 46, 35 (1971).

[8] R. Axelrod and W. D. Hamilton, Science 211, 1390 (1981).

[9] G. C. Williams, Adaptation and Natural Selection (Princeton University Press, NJ, 1966).

[10] D. S. Wilson, The Natural Selection of Populations and Communities (Benjamin/Cummings, Menlo Park, CA, 1980).

[11] R. Donato, J. Phys. I 6, 445 (1996).

[12] R. Donato, L. Peliti, and M. Serva, Theor. Biosci. 116, 309 (1997).

[13] A. T. C. Silva and J. F. Fontanari, Eur. Phys. J. B 7, 385 (1999).

[14] R. Heinsohn and C. Packer, Science 269, 1260 (1995).

[15] J. Maynard Smith, Am. Nat. 99, 59 (1965).

[16] C. T. Bergstrom and M. Lachmann, Anim. Behav. 61, 535 (2001).
[17] P. E. Turner and L. Chao, Nature (London) 398, 441 (1999).

[18] M. Milinski, Nature (London) 325, 433 (1987).

[19] D. Heg and R. van Treuren, Nature (London) 391, 687 (1998).

[20] A. S. Grutter, Nature (London) 398, 672 (1999).

[21] J. Hofbauer and K. Sigmund, Evolutionary Games and Population Dynamics (Cambridge University Press, Cambridge, 1998).

[22] M. Nowak and K. Sigmund, Nature (London) 364, 56 (1993).

[23] R. Ferrière and R. E. Michod, Am. Nat. 147, 692 (1996).

[24] M. Doebeli, A. Blarer, and M. Ackermann, Proc. Natl. Acad. Sci. U.S.A. 94, 5167 (1997).

[25] M.A. Nowak and R. M. May, Nature (London) 246, 15 (1992).

[26] M. A. Nowak and R. M. May, Int. J. Bifurcation Chaos Appl. Sci. Eng. 3, 35 (1993).

[27] M. A. Nowak, S. Bonhoeffer, and R. M. May, Proc. Natl. Acad. Sci. U.S.A. 91, 4877 (1994).

[28] T. Killingback and M. Doebeli, Proc. R. Soc. London, Ser. B 263, 1135 (1996).

[29] T. Killingback and M. Doebeli, J. Theor. Biol. 191, 335 (1998).

[30] K. Lindgren and M. G. Nordahl, Physica D 75, 292 (1994).

[31] K. Brauchli, T. Killingback, and M. Doebeli, J. Theor. Biol. 200, 405 (1999).

[32] D. J. Watts and S. H. Strogatz, Nature (London) 393, 440 (1998). 
[33] G. Abramson and M. Kuperman, Phys. Rev. E 63, 030901 (2001).

[34] M. A. Nowak, S. Bonhoeffer, and R. M. May, Int. J. Bifurcation Chaos Appl. Sci. Eng. 4, 33 (1994).

[35] G. Szabó and C. Töke, Phys. Rev. E 58, 69 (1998).

[36] J. R. N. Chiappin and M. J. de Oliveira, Phys. Rev. E 59, 6419 (1999).

[37] G. Szabó, T. Antal, P. Szabó, and M. Droz, Phys. Rev. E 62, 1095 (2000).

[38] D. Stauffer and A. Aharony, Introduction to Percolation Theory (Taylor \& Francis, London, 1994).
[39] B. Derrida, A. J. Bray, and C. Godrèche, J. Phys. A 27, L357 (1994).

[40] A. J. Bray, B. Derrida, and C. Godrèche, Europhys. Lett. 27, 175 (1994).

[41] D. Stauffer, J. Phys. A 27, 5029 (1994).

[42] S. N. Majumdar, Curr. Sci. 77, 370 (1999).

[43] B. Derrida, P. M. C. de Oliveira, and D. Stauffer, Physica A 224, 604 (1996).

[44] C. M. Newman and D. L. Stein, Phys. Rev. Lett. 82, 3944 (1999).

[45] S. Jain, Phys. Rev. E 60, R2445 (1999). 